\title{
A!
}

This is an electronic reprint of the original article.

This reprint may differ from the original in pagination and typographic detail.

Ptitcyn, Grigorii A.; Mirmoosa, Mohammad S.; Asadchy, Viktar S.; Tretyakov, Sergei A.

\section{Time-modulated reactive elements for control of electromagnetic energy}

Published in:

2019 URSI International Symposium on Electromagnetic Theory, EMTS 2019

DOI:

10.23919/URSI-EMTS.2019.8931443

Published: 01/05/2019

Document Version

Peer reviewed version

Please cite the original version:

Ptitcyn, G. A., Mirmoosa, M. S., Asadchy, V. S., \& Tretyakov, S. A. (2019). Time-modulated reactive elements for control of electromagnetic energy. In 2019 URSI International Symposium on Electromagnetic Theory, EMTS 2019 [8931443] IEEE. https://doi.org/10.23919/URSI-EMTS.2019.8931443

This material is protected by copyright and other intellectual property rights, and duplication or sale of all or part of any of the repository collections is not permitted, except that material may be duplicated by you for your research use or educational purposes in electronic or print form. You must obtain permission for any other use. Electronic or print copies may not be offered, whether for sale or otherwise to anyone who is not an authorised user. 


\title{
Time-Modulated Reactive Elements for Control of Electromagnetic Energy
}

\author{
Grigorii A. Ptitcyn, Mohammad S. Mirmoosa, Viktar S. Asadchy and Sergei A. Tretyakov \\ Department of Electronics and Nanoengineering, Aalto University, P.O. Box 15500, FI-00076 Aalto, Finland
}

\begin{abstract}
Accumulation of electromagnetic field energy in classical passive structures such as resonators and reactive circuit elements is limited by the amplitude of time-harmonic external sources. In the steady-state regime, all incident power which is not absorbed is fully re-radiated (reflected), and the time-averaged stored energy is constant, even though the external source continuously supplies energy. Recently, we have shown that lossless objects can continuously accumulate energy if their properties are properly modulated in time. Here, we discuss this possibility and study means to release this accumulated energy in the desired moment or moments of time in form of pulses of the desired shape. Furthermore, we discuss a possibility of keeping the energy inside the load, turning off the incident wave and changing the modulation function of the load. Finally, instead of a single time-reactive element we consider a parallel time-varying $L C$ circuit, that gives the same functionality, in addition allowing to engineer currents flowing through the load circuit elements.
\end{abstract}

\section{Introduction}

The property of accumulating and releasing of energy without dissipation makes an efficient battery. Electromagnetic field energy can be stored in form of evanescent fields or standing waves inside or near various objects, for example, in cavity resonators, in low-loss metasurfaces, and in reactive circuit elements (capacitors and inductors). If we consider reactive elements connected to a time-harmonic source, then the energy stored inside them depends on the current $(i(t))$ and voltage $(v(t))$ provided by the source, and on the element parameters: capacitance $(C)$ and inductance $(L)$. The stored energy is given by the equation

$$
W_{\mathrm{e}}(t)=C \frac{v(t)^{2}}{2}, \quad W_{\mathrm{m}}(t)=L \frac{i(t)^{2}}{2} .
$$

After the transition period, when the steady-state regime is established, the time-averaged stored energy does not grow and all incident power is fully reflected back to the source. This is a classical limitation which is valid for stationary reactive elements connected to time-harmonic sources. It is possible to overcome it by modulating the signal sent by the source. This approach was realized in Ref. [1]: exponentially growing wave was virtually absorbed inside a dielectric slab and released after stopping the energy pump. However, modifying the source is not always an option, and in this case there is no control over the shape of the released signal. Another way to store energy is to modulate the load reactance in time [2]. This way one can eliminate reflection although there is no dissipation. Since the load is reactive and energy cannot be absorbed, all the incident energy is stored. Here, we show that using time-varying load it is possible to control reflected waves, meaning that aside from unlimited accumulation of electromagnetic energy it is also possible to release the stored energy in form of an arbitrary shaped pulse.

\section{Accumulation of energy by time- modulated reactive elements}

Let us consider a time-varying reactive element, for instance inductance. Voltage on such inductance reads

$$
v_{\mathrm{L}}(t)=L(t) \frac{d i(t)}{d t}+\frac{d L(t)}{d t} i_{\mathrm{L}}(t)
$$

where $v_{\mathrm{L}}(t)$ and $i_{\mathrm{L}}(t)$ denote the instantaneous voltage and current, respectively. For conventional stationary inductor the second term in Eq. (2) is zero. However, for an inductance that changes in time, voltage across it depends on the time derivative of $L(t)$. Moreover, the second term in Eq. (2) resembles Ohm's law which relates voltage with current through a coefficient $\frac{d L(t)}{d t}$, meaning that this derivative can be considered as a positive or negative resistance. In other words, time-varying inductance either produces or absorbs energy. As soon as the inductor is lossless, this energy cannot be absorbed by the element itself, it can be either accumulated in the reactive element or in the modulating device (assuming that it is also lossless).

Let us study time-varying reactive elements on the example of a transmission line terminated by a time-modulated inductance, as illustrated in Fig. 1(a). In order to exploit virtual absorption in time-varying reactive elements let us find the required modulation function that cancels reflection from the load. For convenience, voltage on the inductance can be split into the incident voltage $v^{+}(t)$ and the reflected 


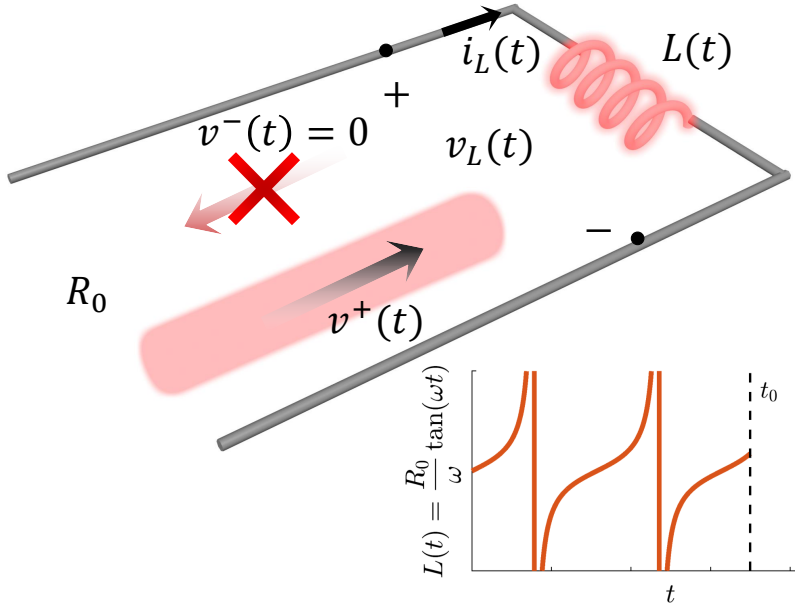

(a)

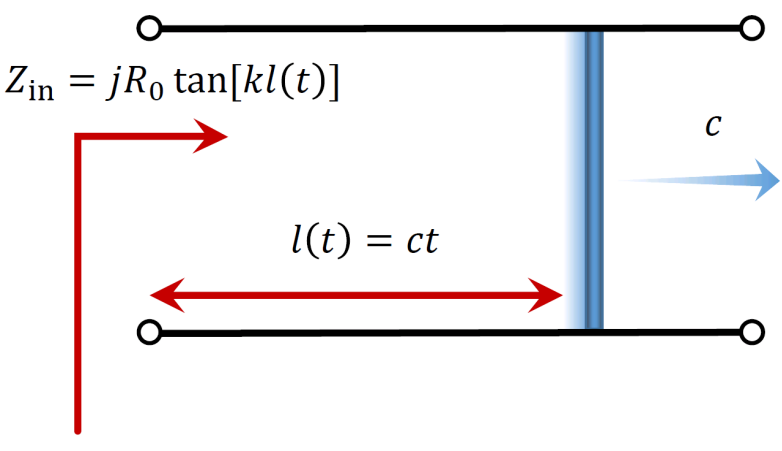

(b)

Figure 1. (a) - Transmission line terminated by a time-dependent inductance which virtually absorbs the incoming energy. (b) - Conceptual realization of the time-dependent inductance by a short-circuited line whose length extends with a constant velocity.

voltage $v^{-}(t)$. This allows us to express the voltage on the inductance and current through it as

$$
v_{\mathrm{L}}(t)=v^{+}(t)+v^{-}(t), \quad i_{\mathrm{L}}(t)=\frac{v^{+}(t)-v^{-}(t)}{R_{0}},
$$

where $R_{0}$ represents the characteristic impedance of the transmission line. Substituting Eq. (2) into Eq. (3) we get a general formula for the incident and reflected waves:

$$
\begin{aligned}
& L(t) \frac{d v^{-}(t)}{d t}+\left[\frac{d L(t)}{d t}+R_{0}\right] v^{-}(t)= \\
& L(t) \frac{d v^{+}(t)}{d t}+\left[\frac{d L(t)}{d t}-R_{0}\right] v^{+}(t) .
\end{aligned}
$$

Assuming a time-harmonic incident wave $v^{+}(t)=$ $A \cos (\omega t)$, and demanding zero reflection $v^{-}(t)=0$, it is possible to solve Eq. (4) and find the required function for $L(t)$. The solution reads

$$
L(t)=\frac{R_{0}}{\omega} \tan (\omega t) .
$$

An inductance modulated according to Eq. (5) eliminates the reflected wave, meaning that it virtually absorbs all the input energy. For better understanding let us draw an analogy between a transmission line terminated with such timevarying load and a short-circuited transmission line. The classical formula for the input reactance of a short-circuited transmission line reads:

$$
Z_{\text {in }}=j R_{0} \tan \left(\frac{\omega}{c} l\right)
$$

where $c$ is the phase velocity and $l$ represents the length of the line. Instead of a constant length $l$ let us now consider a short-circuited line whose length is linearly increasing with constant velocity $c$. The input impedance of such line is $Z_{\text {in }}=j R_{0} \tan (w t)$ (see Fig. 1(b)), which is exactly equal to the impedance of the time-modulated inductance,
$Z_{\text {in }}=j \omega L(t)$. It becomes clear that inductance modulated according to Eq. (5) mimics a short-circuited transmission line of linearly increasing length. We see that in this conceptual scenario the reason for having no reflection from a lossless load is that the incident wave never reaches the reflecting termination, since the short is moving away from the input port with the same velocity as the phase front of the incident wave. Thus, varying the load inductance as prescribed by Eq. (5) for enough long time one can accumulate theoretically unlimited field energy in the reactive load.

\section{Controlling the release of energy}

\subsection{Engineering the shape of output pulse}

Accumulation of energy with no loss is also possible controlling the source [1], however, such approach does not provide any control on how the stored energy will be released. In our case it is possible to stop modulation of the load and the energy will be slowly released, but it is possible to change the modulation regime and engineer the shape of the released pulse. One of the possibilities is to modulate the load as an exponentially decaying function of time. We assume that starting from $t=0$ the time-varying inductance was illuminated by a time-harmonic wave, and the inductance was modulated according to Eq. (5) till the moment $t_{0}=2.5 \mathrm{~s}$. During this period of time, incident energy has been accumulated in the load. Starting from $t=t_{0}$ the incident wave is kept the same, but the load reactance is modulated as an exponentially decaying function:

$$
L(t)= \begin{cases}\frac{R_{0}}{\omega} \tan (\omega t), & t<t_{0}, \\ \frac{R_{0}}{\omega} \tan \left(\omega t_{0}\right) \exp \left(-\alpha\left(t-t_{0}\right)\right), & t>t_{0},\end{cases}
$$

where parameter determines the inductance decay rate. Figure 2(a) graphically represents the varying inductance defined by Eq. (7). The reflected voltage is presented in 


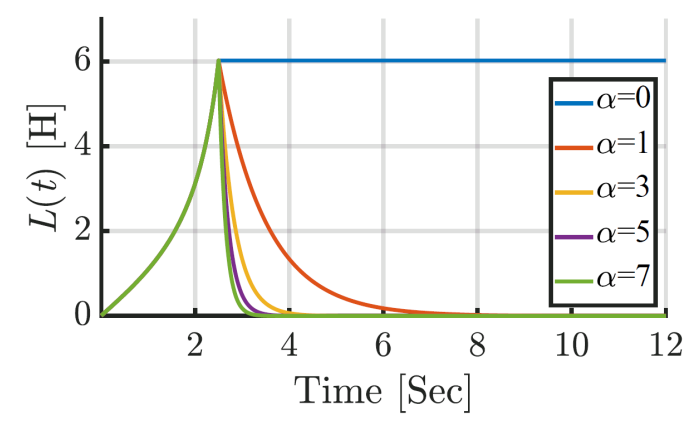

(a)

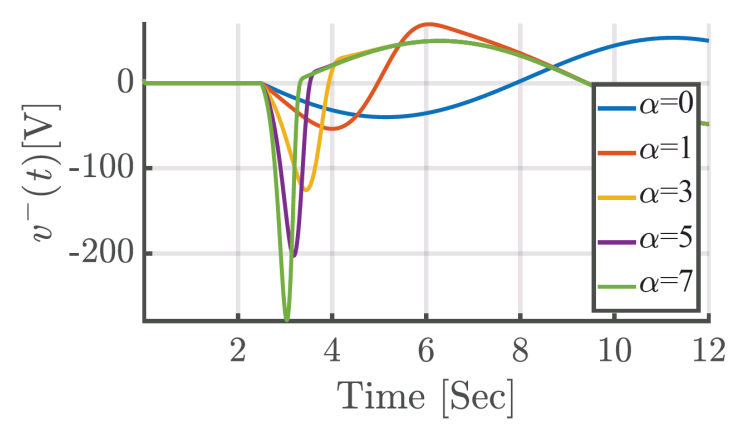

(b)

Figure 2. (a) - Modulation functions of inductance, for different sample values of $\alpha$, that control the shape of the output pulse. (b) - Reflected voltage in form of pulses.

Fig. 2(b). It is clear that changing $\alpha$ allows us to shape the amplitude and width of the output pulse.

\subsection{Forming a delta-function pulse}

We can envisage another way for releasing energy, getting back to the conceptual scenario with a moving short. The short that moves with the phase velocity $c$ reflects nothing. Now let us consider a short that moves backwards after some moment of time $t_{0}$. It means that initially increasing length of the transmission line starts to reduce after moment $t_{0}$. In this case at $t=2 t_{0}$ the length of the transmission line is zero and all the energy is released. However, most importantly, the energy is released in form of a delta-function pulse, since all reflected waves propagate together with the short and arrive to the output simultaneously. In order to realize it, the modulation function should be mirrored with respect to moment $t_{0}$ :

$$
L(t)= \begin{cases}\frac{R_{0}}{\omega} \tan (\omega t), & t<t_{0} \\ \frac{R_{0}}{\omega} \tan \left(2 \omega t_{0}-\omega t\right), & t>t_{0} .\end{cases}
$$

Figure 3(a) shows the time-dependent inductance that generates two output pulses. The function is periodic with the period equal to the period of the incident wave $\left(T_{0}=2 \pi / \omega\right)$ and consists of three regions: $0<t<t_{0}$ when the energy is pumped into the system with zero reflection $\left(t_{0}=2.5 \mathrm{~s}\right.$ in this example), $t_{0}<t<2 t_{0}$ when the time-modulation function is reversed in order to release all the energy by the moment $2 t_{0}$, and $2 t_{0}<t<T_{0}$ when the system relaxes and

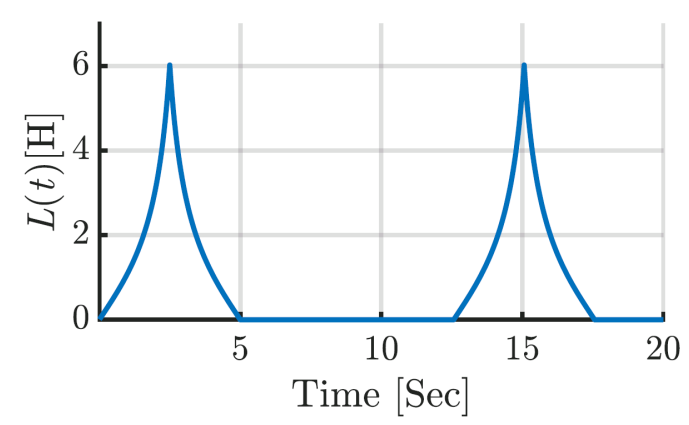

(a)

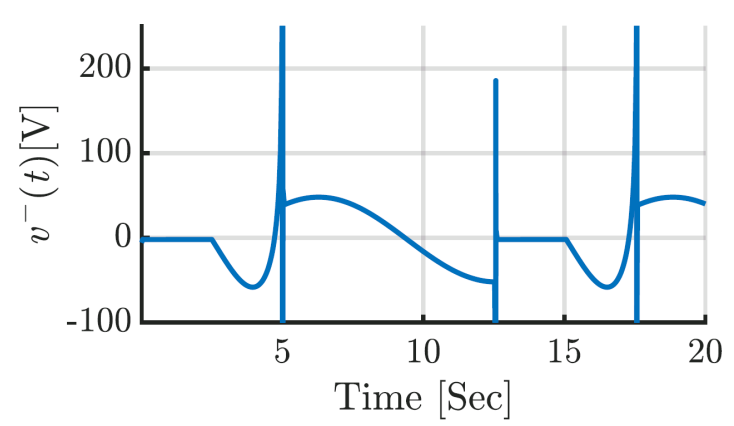

(b)

Figure 3. (a) - Modulation function for inductance that realizes pulse generation. (b) - Reflected voltage from modulated load that exhibit formed short pulses at $2 t_{0}(5 \mathrm{~s})$ and $T_{0}+2 t_{0}(17.6 \mathrm{~s})$.

$L(t)$ is constant and very small. Reflected voltage for such modulation is presented in Fig. 3(b), and we see that indeed at moment $2 t_{0}$ there is huge reflection. At moments $t=0$ and $t=T_{0}$ there is small reflection that occurs because of transitions, nevertheless these reflections decay fast.

In the analogy with a moving short illustrated in Fig. 1(b), there is no need to physically move the short. Conceptually, it is possible to periodically load a transmission line with switches which can be closed at the appropriate time moments [2]. In this scenario the output pulse is formed by a set of periodic pulses that propagate along the line. Initially, all the switches are open and the signal propagates without reflections. When the leading pulse arrives to the last switch, it closes and therefore the pulse is reflected. This reflected pulse and the second incoming pulse approach the second last switch from the opposite directions and at time moment when they meet, the second switch closes, thus forming a reflected pulse of doubled amplitude. It is clear that each next switch increases the amplitude of the reflected pulse, meaning that the energy at the output is proportional to the squared number of pulses entering the transmission. However, the total energy entered the transmission line is proportional to the number of pulses. It means that extra energy comes from the device which controls the switches.

From another perspective, in order to achieve zero reflection, the requirement that has to be fulfilled is the 


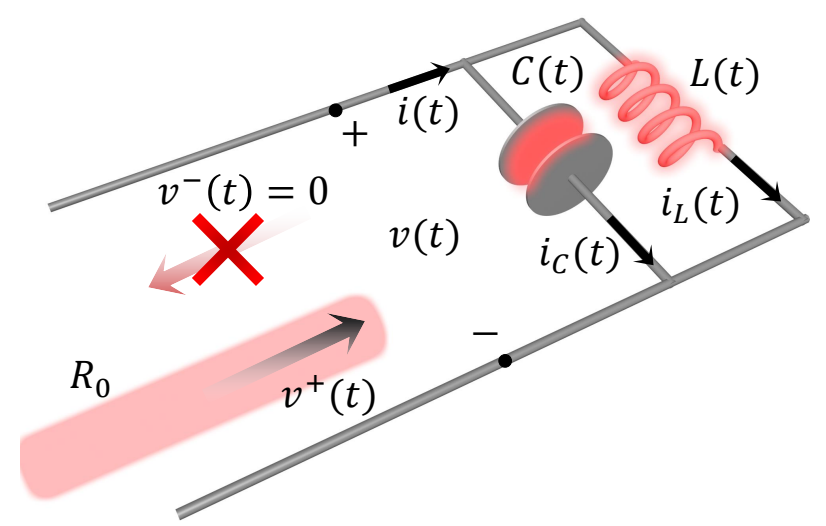

(a)

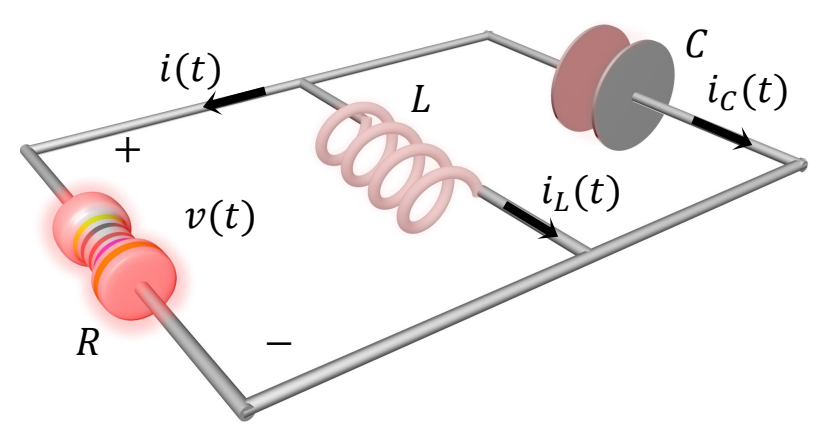

(b)

Figure 4. (a) - Transmission line terminated by a parallel $L(t) C(t)$-circuit. (b) - Corresponding $R L C$-circuit after stopping modulation.

impedance matching, meaning that a single time-varying element given by Eq. (5) can be replaced by a combination of conventional elements that give the same impedance. In order to do it one can expand periodical function in Eq. (5) into Fourier series and then realize components of the series using harmonically pumped mixers and filters [2].

\subsection{Energy keeping regime}

Modulating reactive load makes possible to cancel the reflection and virtually absorb the incident energy. It also allows us to engineer the shape of released pulse. However, there is an additional functionality that is vital for energy storing applications. By changing modulation, it is possible to realize energy keeping regime. In this case Eq. (4) can be solved assuming zero reflection at all moments of time and exponentially decaying incident wave after some moment of time $t_{0}$. In this case the time-varying load keeps the energy as long as required and afterwords the energy can be released. Surely, pumping circuit spends energy on modulation, but finally all the energy can be released [2].

\section{Engineering currents in the branches of parallel $L C$ circuit}

Solution for a single time-varying element, that cancels the reflection, leaves no freedom for electric current flowing through the element. It is defined by the characteristic impedance of the transmission line and the amplitude of the incident wave. However, replacing a single reactive element via a circuit composed of two elements (see Fig. 4(a)) allows us to choose functions for currents flowing through the components. Modulating both of these elements allows us to engineer time-dependences of the currents in the branches with one restriction on the sum of the currents, which should equal $i(t)=A \cos (\omega t) / R_{0}$ in order to ensure zero reflection. Currents in the branches uniquely define the corresponding functions for the reactive elements, which makes possible to find simple and realizable modulation functions for reactive elements.

\section{Conclusion}

In conclusion, we have developed our results on timevariant reactive elements which can continuously accumulate energy from conventional external time-harmonic sources, without any reflections of the incident power, focusing on the regime of energy release. We have found the required time dependences of reactive elements and discussed possible realizations as time-space modulated transmission lines and mixer circuits. We have shown that modulated reactive load as exponentially decaying inductance provides control over the shape of the released pulse. Moreover, we also discussed a possibility of realizing energy keeping regime in the absence of incident waves. Indeed, exploiting time-varying reactive loads offers a vast range of possibilities, which become possible if the modulation function is not limited to time-harmonic and even periodical functions. Although the study has been made for, probably, the simplest example of a time-varying inductance, the results can be directly applied to waves incident on lossless time-modulated boundaries, since they can be modelled as bulk loads in an equivalent transmission line.

\section{References}

[1] D. G. Baranov, A. Krasnok, and A. Alù, "Coherent virtual absorption based on complex zero excitation for ideal light capturing," Optica, 4, December 2017, pp. 1457-1461, doi: 10.1364/OPTICA.4.001457.

[2] M. S. Mirmoosa, G. A. Ptitcyn, V. S. Asadchy, and S. A. Tretyakov, "Unlimited accumulation of electromagnetic energy using timevarying reactive elements," Preprint, February 2018, arxiv.org/abs/1802.07719. 21.02.2018 